\title{
Influencia publicitaria por redes sociales en las compras de consumidores jóvenes del Distrito Central, 2016
}

\author{
Sunee Eiamwasant, Berlín Cáceres, Bessy Cruz ${ }^{1}$
}

\section{RESUMEN}

El objetivo de la investigación fue evaluar la influencia de la publicidad a través de redes sociales en el comportamiento de compras de los jóvenes indicados inicialmente; basándose en el aumento del uso del Internet y de redes sociales.

Se investigó el uso que le dan a las mismas y si las empresas las utilizan para influenciar, persuadir, informar y motivar a su mercado meta a adquirir productos y servicios. Se utilizó el método de investigación objetivista, con un diseño mixto (cualitativo y cuantitativo), utilizando como técnica cualitativa el grupo focal y del diseño cuantitativo la encuesta y la observación estructurada; entrevistando un total de 385 usuarios de redes sociales, distribuidos $52 \%$ hombres y $48 \%$ mujeres.

Concluimos que los usuarios de redes sociales las utilizan un 93\% diariamente; $90 \%$ para socializar, 34\% para informarse de nuevos productos, $29 \%$ para realizar compras y $23 \%$ con fines laborales. Denotando que la publicidad que más se destaca en este medio, es la publicidad de marca; misma que se centra en el desarrollo de la identidad e imagen corporativa y el producto, obteniendo que $81 \%$ de los entrevistados manifiesta que es la que más llama su atención y es utilizada por las empresas para reforzar la experiencia de compra.

Los empresarios participantes en el grupo focal indicaron que hacen uso de las siguientes redes sociales: Facebook, Instagram y Twitter para realizar publicidad de sus productos.

Palabras clave: Publicidad por internet, Internet, redes sociales, comportamiento del consumidor

\footnotetext{
${ }^{1}$ Beneficiarios de una beca básica de la DICYP. Profesores de la Carrera de Mercadotecnia, Facultad de Ciencias Económicas, Administrativas y Contables, UNAH: lopez.sunee@unah.edu.hn; bcaceres@unah.edu.hn; bessy.cruz@unah.edu.hn
} 


\section{ABSTRACT}

The objective of this research was to evaluate the influence of advertising through social networks on the purchasing behavior of young people based on the increased use of the Internet and social networks.

We investigated the use they give to social networks and if the companies use them to influence, persuade, inform and motivate their target to buy products and services. The objectivist research method was used, with a mixed design (qualitative and quantitative), using as a qualitative technique the focus group and the quantitative design survey and structured observation; interviewing a total of 385 users of social networ$\mathrm{ks}$, distributed $52 \%$ men and $48 \%$ women.

In conclusion the social network users use them $93 \%$ daily; $90 \%$ to socialize, $34 \%$ to learn about new products, $29 \%$ to make purchases and $23 \%$ for work purposes. Denoting that the advertising that stands out the most in this medium is brand advertising; same that focuses on the development of the identity and corporate image and the product, obtaining that $81 \%$ of the interviewees state that it is the one that catches their attention and is used by companies to reinforce the shopping experience.

The businessmen participating in the focus group indicated that they use the following social networks: Facebook, Instagram and Twitter to advertise their products.

Keywords: : Internet advertising, Internet, social network, consumer behavior. 


\section{INTRODUCCIÓN}

Este artículo presenta los principales resultados logrados en el proceso de investigación sobre el tema que aborda la influencia de la publicidad por redes sociales en las decisiones de compra de los consumidores jóvenes en el contexto del Distrito Central, durante el período comprendido de febrero a mayo, 2016. El objetivo del estudio es identificar cuáles son las redes sociales que más utilizan las personas de las edades entre los 18 a 32 años, qué productos son los que más buscan, tiempo promedio de visita a las redes sociales y los motivos que predominan para acceder a una red social determinada.

La principal justificación para realizar el estudio es que actualmente el uso de las redes sociales por parte de las empresas es casi fundamental, ya que permite que éstas eleven su potencial al llegar a segmentos de mercado que podrían ser inalcanzables para la misma, ya sea por su tamaño, geografía y estructura, permitiendo que la web sea un enlace con sus clientes actuales y potenciales, así como para los usuarios poder incrementar la oferta de productos nuevos o mejorados a los que pueden tener acceso.

Las empresas requieren mantenerse comunicadas con sus clientes, informar acerca de sus productos o simplemente recordarles que existen y que son una opción para satisfacer sus necesidades y deseos, por lo que el uso de esta herramienta nos provee un mundo de oportunidades que van desde informar a través del uso de la publicidad hasta obtener ventajas competitivas al facilitar la creación de mercados y de nuevos segmentos para la empresa.

Al analizar el uso de redes sociales tanto desde el punto de vista del consumidor como del empresario anunciante, la investigación se enfoca en:

1. Identificar los tipos de productos más buscados por el usuario de redes sociales en las edades de 18 a 32 años.

2. Determinar la frecuencia de uso de las redes sociales por parte de los consumidores jóvenes de estudio para conocer nuevos productos.

3. Identificar qué redes sociales tienen más influencia en los consumidores jóvenes para tomar la decisión de adquirir productos. 


\section{METODOLOGÍA}

El diseño de la investigación constituye el plan o estrategia que se desarrolla para obtener la información requerida en una investigación (Roberto Hernández Sampieri, 2010). En este estudio se aplicará el método general de investigación aplicando un enfoque objetivista a través del análisis documental y la aplicación de trabajo. Según (Muñoz Alonso, 2011, pág. 219) algo es objetivo cuando existe realmente 0 cuando es parte de un objeto real. Aunado al planteamiento de la implementación del método de investigación objetiva, para el desarrollo del presente estudio se aplicará un diseño de investigación mixto, ya que se desarrollará tanto exploratoria (cualitativa) como concluyente (cuantitativa) - descriptiva - transversal simple.

Los métodos mixtos representan un conjunto de procesos sistemáticos, empíricos y críticos de investigación e implican la recolección y el análisis de datos cuantitativos y cualitativos, así como su integración y discusión conjunta, para realizar inferencias producto de toda la información recabada (metainferencias) y lograr un mayor entendimiento del fenómeno bajo estudio. (Sampieri, Fernández Collado, \& Baptista Lucio, 2014)

La población o muestra de estudio estuvo constituida por hombres y mujeres jóvenes en edades de 18 a 32 años usuarios de redes sociales residentes en el Distrito Central, esto debido a que en la segmentación por generaciones, este rango de edad presenta una conducta influenciada por la publicidad al momento de realizar sus compras; tomando también en cuenta que según una medición de la empresa brasileña de mercadeo y medición de audiencias Ibope Media, en los últimos tres meses del año 2011, al menos 1.2 millones de hondureños en las principales ciudades se habían conectado a Internet. De ellos, 726,696 lo hicieron en la capital, mientras 476,114 entraron a la red en San Pedro Sula.

De los cibernautas, $52 \%$ son hombres y el restante $48 \%$ mujeres; además, $46 \%$ pertenecen a la clase media, $30 \%$ a la alta, $22 \%$ a la clase baja y el $2 \%$ a una clase muy baja.

Otro aspecto equitativo lo representa la edad de los navegadores: $55 \%$ son adultos entre 25 y 32 años y jóvenes $45 \%$ en edades entre 18 y 24 años; se consideró también conocer la opinión de los empresarios que hacen uso de redes sociales para publicar sus marcas, productos y/o servicios como ser: ropa, electrónica, música, área de salud y marca personal. 


\begin{tabular}{|l|c|c|c|}
\hline \multicolumn{1}{|c|}{ Parámetros } & & Edades y Proporción \\
\hline \multicolumn{1}{|c|}{ Sexo } & $\%$ & Muestra Total & De 18 - 24 años 45\% \\
\hline Hombres & 52 & 200 & 90 \\
\hline Mujeres & 48 & 185 & 83 \\
\hline Totales & 100 & 385 & 173 \\
\hline
\end{tabular}

Fuente: Propia

El total de personas a encuestas fue de 385 considerando que la población de Tegucigalpa de acuerdo a los datos estadísticos obtenidos del Censo 2013 a través del Instituto Nacional de Estadísticas y Censos, en la zona urbana de Francisco Morazán la población que utiliza internet es de 451,523 personas en total.

Sample size calculator

1. Confidence level (nivel de confianza): $95 \%$

2. Confidence interval (intervalo de confianza): $+/-5$

3. Population (población): 451523

4. Sample size (tamaño de la muestra): 384

Terminología:

El nivel de confianza le muestra que tan seguro está de sus resultados. Usted puede decir que esta 95\% (o 99\%) seguro de que el verdadero porcentaje de la población se encuentra dentro de su intervalo de confianza. La mayoría de los estudios utilizan el $95 \%$ de nivel de confianza.

El intervalo de confianza es una figura de más o menos que usualmente se reporta en las encuestas de opinión de un periódico o un noticiero de televisión, por lo tanto, cuentan con un margen de error. Por ejemplo, si usted utiliza un intevalo de confianza de 5 , entonces el $23 \%$ de su muestra elige una respuesta particular. Usted puede estar seguro de que si le hubiese preguntado a la población completa, entre $18 \%$ (23-5) y $28 \%(23+5)$, ellos hubiesen elegido esa respuesta.

La población incluye a todos los individuos identificados en el grupo de estudio 
En una primera etapa se establecieron parámetros de cuota para obtener información de la población meta, se identificaron y seleccionaron las técnicas apropiadas para la recolección de datos e información, se realizaron pruebas piloto de los instrumentos y se ajustaron previamente para su aplicación. Dada la naturaleza de la investigación se utilizaron tres técnicas con sus respectivos instrumentos para trabajar con los diferentes actores clave: grupo focal, observación estructurada y la encuesta.

En la segunda etapa se realizó la recolección de datos e información de las fuentes primarias y secundarias, la sesión de grupo focal fue dirigida a empresarios y las encuestas a consumidores usuarios de redes sociales.

En una tercera etapa, se incorporaron sugerencias producto de la realización de un conversatorio tanto con expertos en el uso del marketing digital y estudiantes de la Facultad de Ciencias Administrativas Económicas y Contables de Ciudad Universitaria y los procesos de capacitación a empresarios en el tema; esto como parte de las jornadas de socialización y retroalimentación del informe final.

\section{MARCO TEÓRICO}

\section{Redes sociales en Internet}

Según Larry Weber en su libro Marketing en las redes sociales; define a las redes sociales como "Un lugar en línea donde la gente con intereses comunes puede reunirse para intercambiar pensamientos, comentarios y opiniones, estos sitios incluyen redes sociales como Facebook, MySpaces, Garther, Black Planet, Eons, Linkedin y cientos" (Weber, 2010).

Las tecnologías de Internet avanzan demasiado rápido, para que alcanzaran 50 millones de usuarios (audiencia), a la radio le tomó 38 años, a la televisión 13 años a Internet 4 años a Facebook tan solo 2 años (Roger , William , \& Patton, 2011), para el 2012 existían en Internet aproximadamente 2,267,233,742 usuarios de Internet, que representan el 37\% de la población total (MiniWatts Marketing Group, 2012). (Lopez Salazar, Blanco, Blanco Vidal, Rodriguez, \& Barrera Arias, 2014)

Debido a este crecimiento tan espectacular en el surgimiento de éstas formas y herramientas populares, se pueden mencionar dos principios básicos que han regido 
el comportamiento de las redes como Internet y que, seguramente se mantendrán de esta forma por mucho tiempo, a continuación lo veremos:

"El valor aumenta con el número de los participantes de la red", (Ley de Metcalfe), consiste en decir que si una empresa publicita su marca o servicio en una red de "contactos" mientras más contactos tenga esa red más popular se hará su producto, pero a velocidades increiblemente altas. Por lo tanto, en Internet siempre debe seguir esas "redes" de contactos con fines comunes, aquí las redes sociales, han demostrado un gran potencial. (Weber, 2010)

El otro principio, tiene que ver con la "Economía de Internet", si bien es cierto que las reglas económicas y mercadológicas de la compra-venta en Internet son iguales que en su contraparte "tradicional" o "cara a cara", basta decir que la gran diferencia es que en Internet se puede aumentar el poder de "producción" relativamente rápido, obteniendo resultados casi de inmediato. (Lopez Salazar, Blanco, Blanco Vidal, Rodriguez, \& Barrera Arias, 2014).

\section{Publicidad en redes sociales}

Según Wells, Moriarty y Burnett en su libro Publicidad, Principios y Practicas, definen a la Publicidad como "comunicación persuasiva pagada que utiliza medios masivos e interpersonales asi como otras formas de comunicación interactiva, para llegar a una amplia audiencia y conectar a un patrocinador identificado con el publico meta" (Wells, Moriaty, \& Burnett, 2007).

La publicidad general desempeña 7 funciones basicas:

1. Crea conciencia de productos y marcas.

2. Crea una imagen de marca.

3. Proporciona información de producto y de marca.

4. Persuade a la persona.

5. Brinda incentivos para poner en marcha una acción.

6. Proporciona recordatorios de marca.

7. Refuerza compras y experiencias pasadas de marca.

Según Kevin Sigliano en su guía de la publicidad para redes sociales hace mención que la publicidad directa en medios sociales está creciendo en inversión, teniendo tres activos importantes frente a otras formas de publicidad online. Es óptima tanto para estrategias de branding como promocioales. 
1. Micro segmentación: Los usuarios realizan cientos de interacciones diarias en las redes sociales, muchas de estas interacciones crean opciones de segmentación al instante por tanto, disponemos de un perfil sociodemográfico del "lector" de la red social sin obsolescencia y basado en actos y declaraciones.

2. Difusión orgánica: Uno de los principales activos de la publicidad en medios sociales es que los usuarios que la ven pueden interactuar con ella. No sólo "clicar", cada interacción puede provocar una nueva redifusión del contenido del anuncio, desde ese momento ya sin costo.

3. Familiaridad: En el propio formato del anuncio podemos hacer que aparezca la imagen de un contacto en la red social de quien está viendo el anuncio.

\section{RESULTADOS}

Los principales hallazgos de la investigación se detallan a continuación, estos se presentan de acuerdo a las tres técnicas de investigación aplicadas: grupo focal, encuesta y observación estructurada.

\section{Figura 1. Rubros de las empresas}

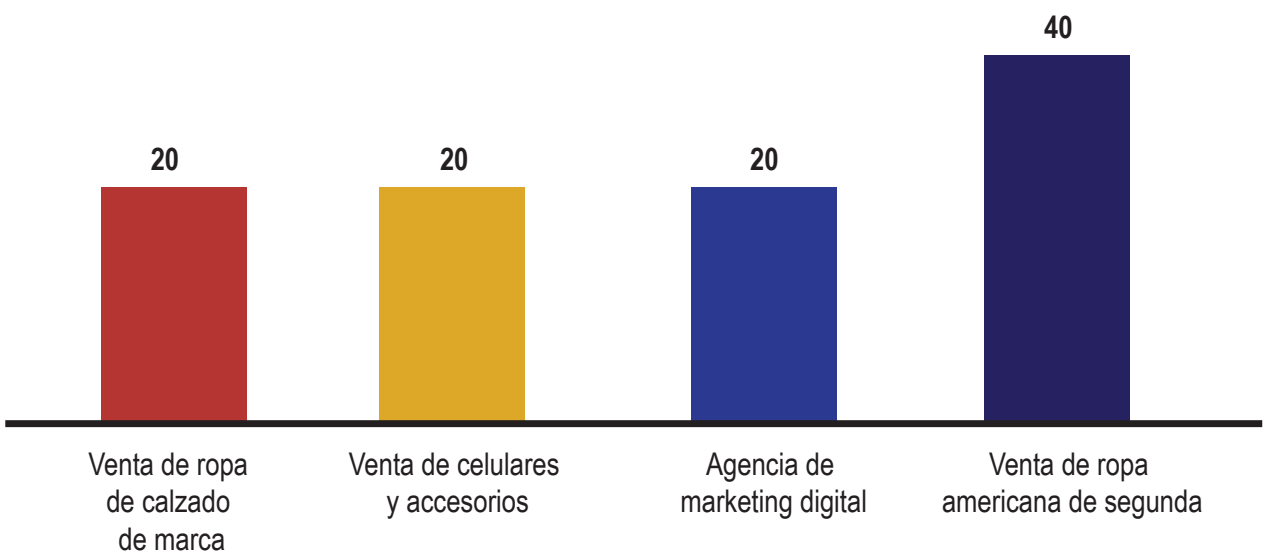

Fuente: Elaboración propia, resultados de la investigación

De los empresarios que asistieron al grupo focal podemos observar que el $80 \%$ ofrece bienes y el $20 \%$ servicios.

Un aspecto relevante es que el $40 \%$ ofrece ropa usada un aspecto bastante particular por el tipo de producto. 
Figura 2. Redes sociales utilizada

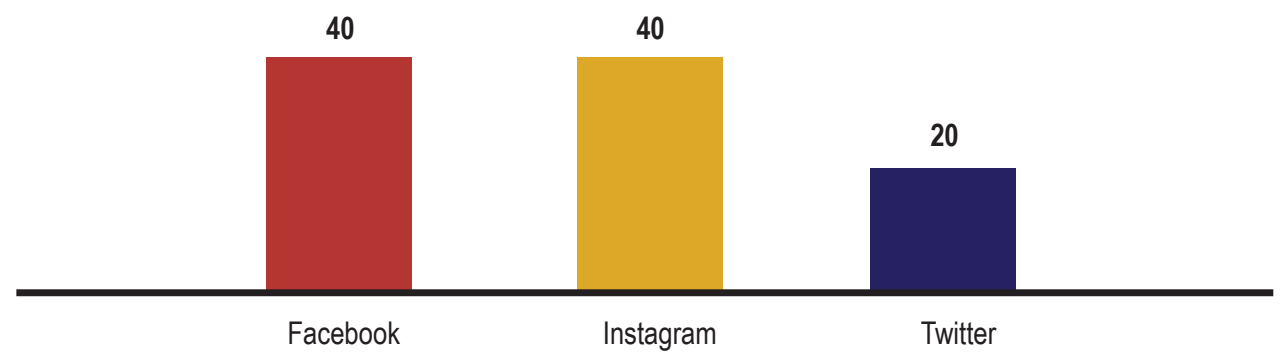

Fuente: Elaboración propia, resultados de la investigación

Las redes sociales más utilizadas son Facebook, Instagram y Twitter por parte de los empresarios.

Cabe mencionar que indicaron utilizar estas redes sociales porque son las que los usuarios más utilizan según su experiencia, agregando el uso del WhatsApp como herramienta complementaria para comunicación.

Según estudio realizado por la agencia de marketing digital Web Empresa 20, el ranking redes sociales más utilizadas en el mundo al 2015 (Nieto, 2015), la constituyen en primer lugar Facebook con 1,650 millones de usuarios e Instagram ocupa la novena posición, pero los empresarios en Honduras la utilizan en igual proporción que Facebook, es importante destacar que Twitter sigue en uso a pesar de que este último año a nivel internacional se ha visto su caída paulatina, esto debido a la salida de algunos de sus directivos, caída del sistema, cambio de favoritos por "Me gusta"; ocupando según lo investigado la décima posición con 350 millones de usuarios activos.

WhatsApp nació en 2009 y actualmente cuenta con 1,000 millones de usuarios activos y es una herramienta que como se indica anteriormente, es utilizada por los empresarios hondureños como medio de comunicación complementario, permitiendo no solo informar, sino enviar mensajes privados, imágenes, audio y video.

El $40 \%$ de los empresarios publican en las redes sociales cuando tienen nuevos productos y en igual proporción de importancia: a diario, en fechas específicas como ser del 18 al 3 de cada mes basándose en las fechas de pago de sus clientes, realizando entre 2 a 3 publicaciones diarias. 
Figura 3. Frecuencia de publicación.

40

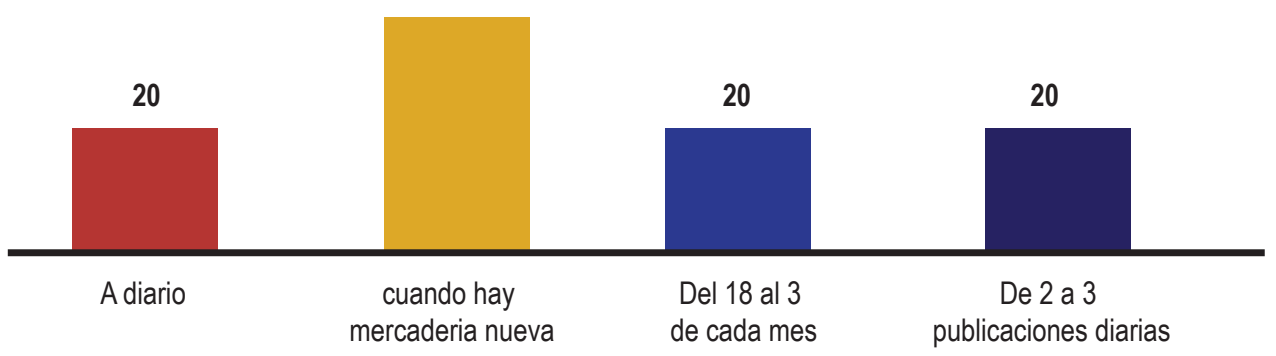

Fuente: Elaboración propia, resultados de la investigación

Principales hallazgos del análisis de las encuestas aplicadas a usuarios de redes sociales

En contraste con las respuestas obtenidas en el grupo focal por parte de los empresarios acerca de las redes sociales que éstos utilizaban para realizar publicidad, se indagó a los jóvenes cuáles eran utilizadas por estos, obteniendo que:

\section{Hallazgo No 1 - Uso de las redes sociales}

Se identificó cuáles redes sociales tienen más influencia en los consumidores jóvenes para tomar la decisión de adquirir productos, dando como resultado lo que muestra en la figura 4:

Figura 4. Uso de redes sociales

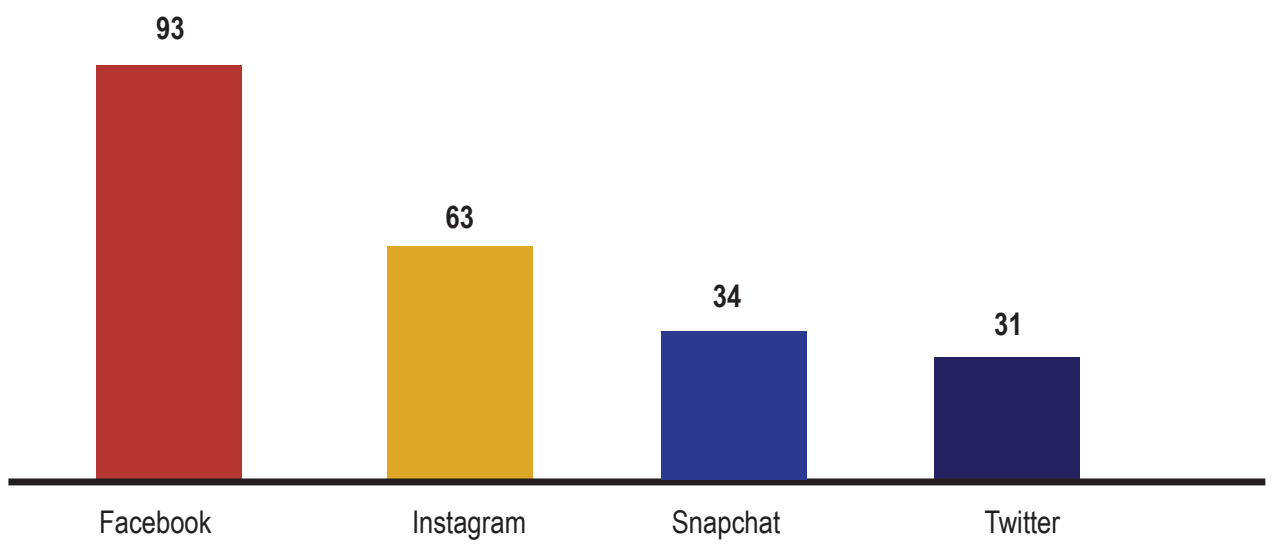

Fuente: Elaboración propia, resultados de la investigación 
La red social de mayor uso por parte de los encuestados es Facebook con un $24 \%$ de participación y en segunda instancia Instagram con un 16\%. Cabe denotar que los usuarios podrían marcar el uso de más de una red social.

Dentro de los objetivos específicos de esta investigación, fue identificar los tipos de productos más buscados por el usuario de redes sociales en las edades de 18 a 32 años y determinar la frecuencia de uso de las redes sociales por parte de los consumidores objeto de estudio para conocer nuevos productos; obteniendo como resultados lo que nos muestran la Figura 5 y 6 respectivamente:

Figura 5. Productos adquiridos a través de redes sociales.

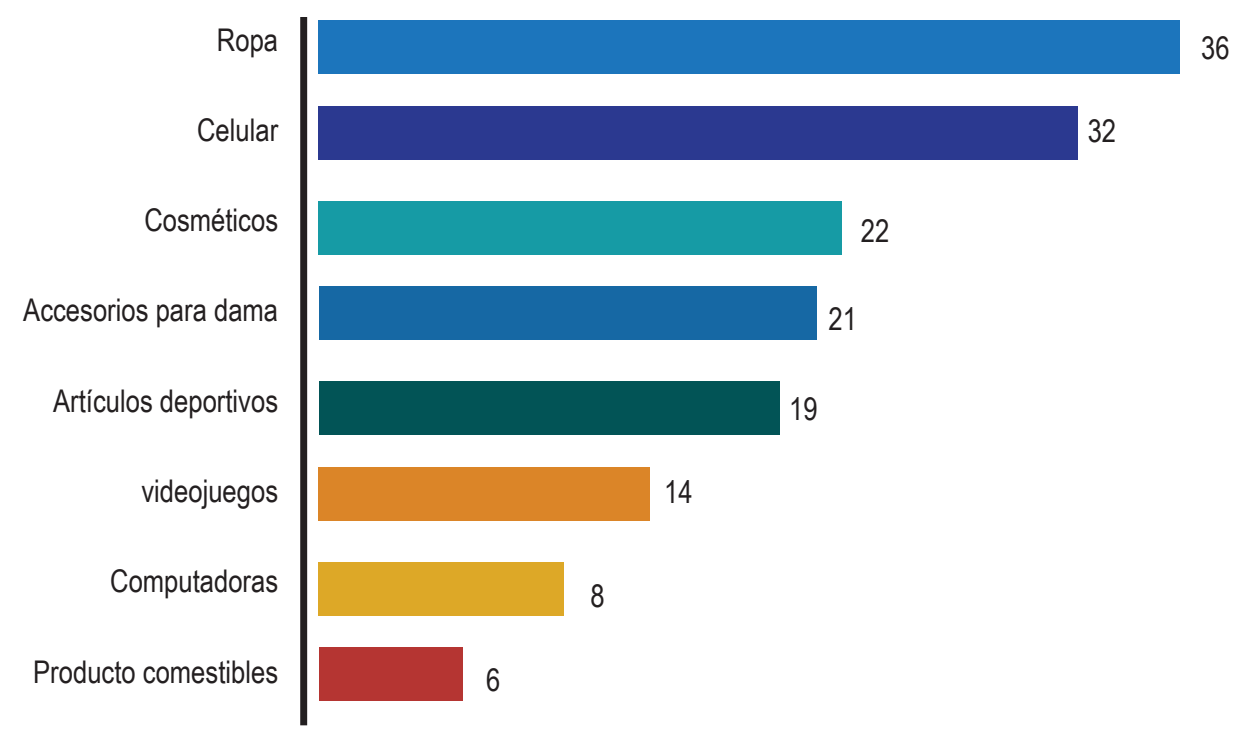

Fuente: Elaboración propia, resultados de la investigación

Como se puede observar, los dos artículos más adquiridos fueron la compra de ropa y compa de aparatos móviles como celulares y lo que menos adquieren los usuarios son productos comestibles.

La frecuencia de uso de las redes sociales es a diario el 93\%, contrastando con lo indicado por parte de los empresarios, al menos el $40 \%$ publica de 2 a 3 veces y lo hacen a diario respectivamente. 
Figura 6. Frecuencia de uso de redes sociales

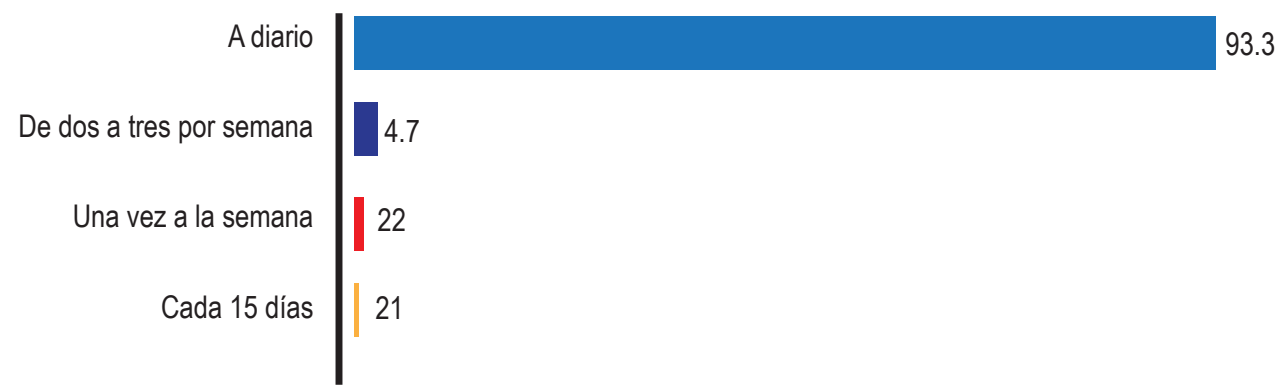

Fuente: Elaboración propia, resultados de la investigación

De igual forma, es importante conocer por qué los usuarios entre los 18 a 32 años específicamente utilizan redes sociales, como lo muestra la figura 8 , el $63 \%$ lo hace para informarse de nuevos productos (34\%) y comprar productos (29\%), en un $90 \%$ las redes sociales se utilizan con el tema de socialización.

\section{Hallazgo N 2 - Razones de uso de las redes sociales}

Figura 7. Motivos de uso de redes sociales

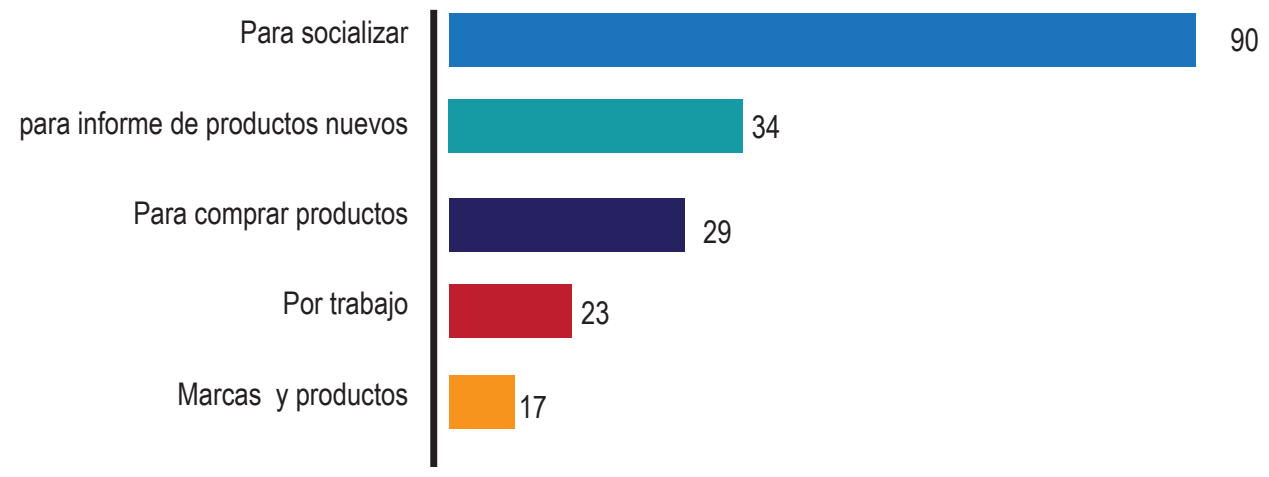

Fuente: Elaboración propia, resultados de la investigación 
Hallazgo $N^{0} 3$ - Interés de la publicidad por redes sociales e influencia de sus grupos de referencia en sus decisiones de compra

Figura 8. Publicidad en redes sociales revisión de la publicidad en redes sociales.

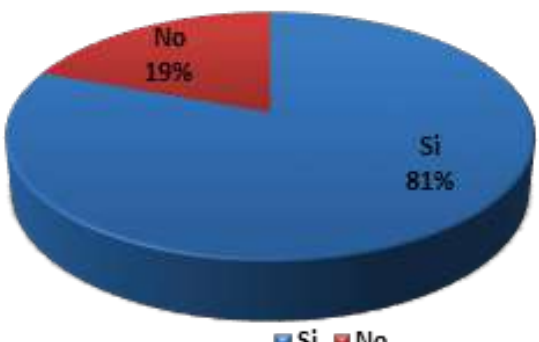

BSi $\mathbf{n}$ No

Fuente: Elaboración propia, resultados de la investigación

Figura 9. Interacción de grupos de referencia en redes sociales en las conversaciones con amigos influyen los siguientes aspectos:

Comentarios sobre precios y calidad

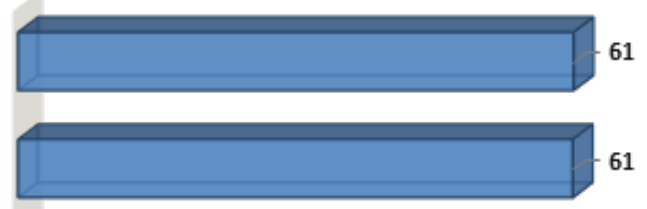

Comentarios sobre la publicidad en las redes sociles

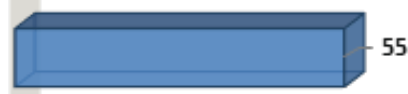

Consejos de moda y marcas de ropa

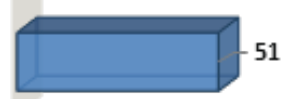

Fuente: Elaboración propia, resultados de la investigación

El $81 \%$ de los encuestados que revisan la publicidad por redes sociales los temas de interacción con sus grupos de referencia a precios y percepción de calidad en un $16 \%$, de igual forma la recomendación de puntos de venta, elemento no menos importante, un $14 \%$ comenta acerca de la publicidad que se transmite a través de este medio. 
Hallazgo $N^{0} 4$ - Uso de redes sociales para informarse de nuevos productos 0 servicios

La aplicación del marketing de búsqueda por parte del consumidor, tal y como lo muestran las Figuras 10 y 11 respectivamente, indican que el 67\% realiza búsqueda de nuevos productos y el $78 \%$ de nuevos productos, sin embargo como pudimos observar en la Figura 5, los entrevistados compran productos tangibles y en menor proporción servicios.

Figura 10. Uso de las redes sociales para la búsqueda de nuevos productos

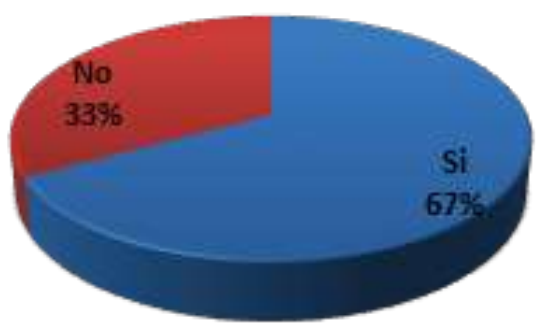

\section{$\mathrm{BSi}$ No}

Fuente: Elaboración propia, resultados de la investigación

Figura 11. Uso de las redes sociales para la búsqueda de nuevos servicios

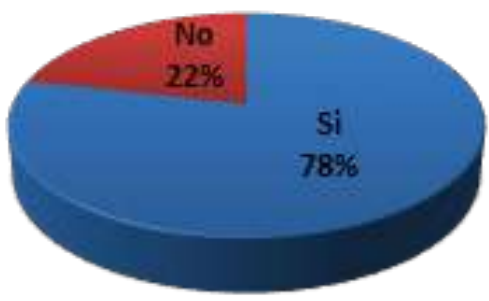

aSi $\operatorname{mo}$

Fuente: Elaboración propia, resultados de la investigación 


\section{Principales hallazgos de la observación estructurada}

Con el objetivo de monitorear el número de publicaciones así como los días que las empresas las realizan, en la Figura 12 podemos observar que algunas empresas, no realizan publicaciones durante el día lunes y los días que considera oportunos son los día viernes con un $53 \%$ y los domingos por la tarde con un $33 \%$ en la jornada vespertina. Asimismo, la Figura 13 nos muestra los usuarios gustan de la publicidad a través de este medio y la califican en mayor proporción el día martes y en igual proporción los días lunes, miércoles, viernes y domingo.

Figura 12. Días de publicación

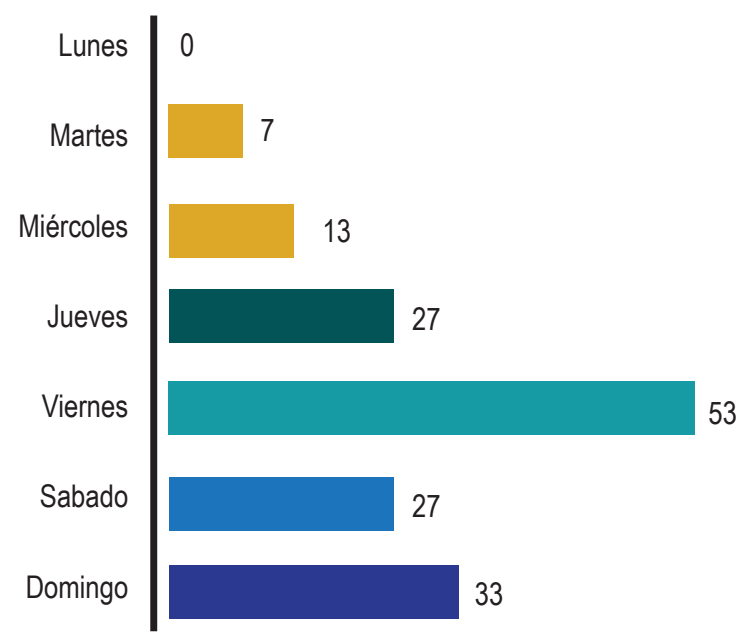

Figura 13. "Me gusta" la publicidad

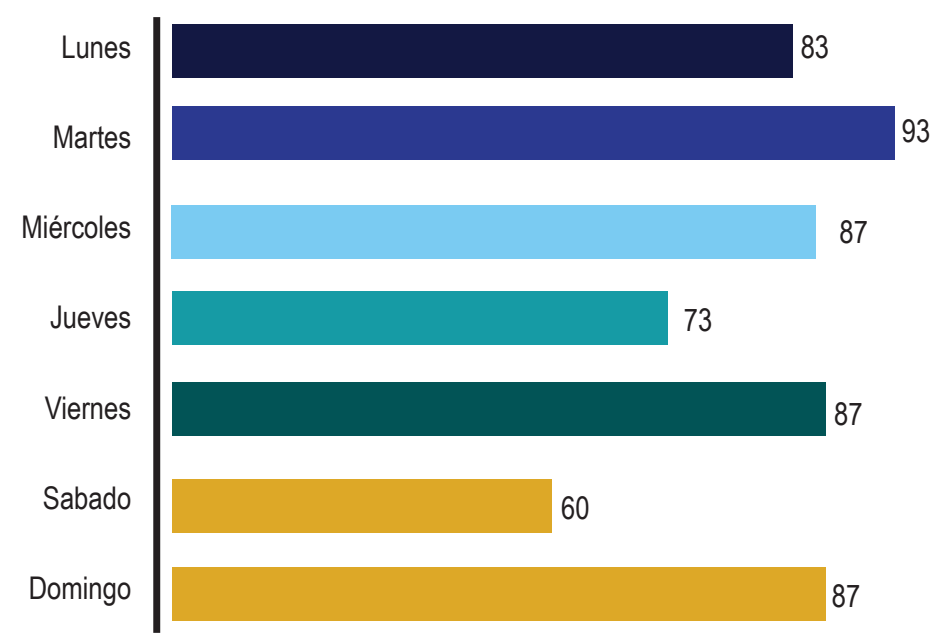

Fuente: Elaboración propia, resultados de la investigación 


\section{CONCLUSIONES}

De acuerdo a la investigación cualitativa y cuantitativa realizadas y con base a los objetivos de investigación se puede concluir lo siguiente:

1. Los tipos de productos más buscados a través de las redes sociales para este segmento de mercado fueron: en primer lugar la ropa, en segunda posición los celulares, los cosméticos en tercer lugar, accesorios para dama en cuarta posición de importancia, artículos deportivos en quinto lugar, los videojuegos en sexto lugar y finalmente los menos buscados y en última posición, productos comestibles.

2. El $93 \%$ de los encuestados utilizan las redes sociales a diario, sin embargo solo un $67 \%$ lo utiliza para la búsqueda de nuevos productos y un $78 \%$ en la búsqueda de nuevos servicios. Importante recalcar que no utilizan las redes sociales con el fin de identificarse con la marca o la adquisición de productos lo hace con el fin de socializar y un modo de entretenimiento.

3. Las redes sociales más utilizadas tanto por los usuarios como las empresas que se realizan publicidad a través de este medio son: Facebook en primer lugar, seguido de Instagram y en tercera posición Twitter. Un elemento importante que agregan las empresas, es la incorporación de la aplicación WhatsApp con el objetivo de tener una interacción más privada con el consumidor, utilizando dicha aplicación para mensajes, imagen y video.

4. Los usuarios de redes sociales gustan de la publicidad a través de este medio, interactúan con sus grupos de referencia haciendo comentarios de la misma, brindando un seguimiento y por ende en algunos casos llevándoles a la acción de compra.

5. El uso de publicidad para informar, persuadir y motivar la acción de compra es utilizada en el Distrito Central a diario por parte de las empresas, realizando entre dos a tres publicaciones diarias.

\section{REFERENCIAS BIBLIOGRÁFICAS}

Lopez Salazar, A., Blanco, M., Blanco Vidal, G., Rodriguez, A. R., \& Barrera Arias, E. (2014). HERRAMIENTAS PARA LA COMPETITIVIDAD DE LA PEQUEÑA EMPRESA EN AMÉRICA LATINA. Mexico: Servicios Académicos Internacionales para eumed.net. Muñoz Alonso, G. (2011). Estructura, metodología y escritura del Trabajo de Fin de Máster. Roberto Hernández Sampieri, C. F. (2010). Metodología de la Investigación. México: McGraw 
Hill.

Roger , F., William , U., \& Patton, B. (2011). Gettin to YES.Negotiating Agreement without Giving in.Penguin Books New York.

Sampieri, R. H., Fernández Collado, C., \& Baptista Lucio, M. (2014). Metdología de la Investigación. México: McGraw Hill.

Sigliano, K., Gonzalez, P. J., Calzada, B., \& Marquez, M. (s.f.). Guia profesional de la publicidad en redes sociales. Bogota: Territorio Creativo.

Treviño, R. (2010). Publicidad. Mexico: McGrawHill.

Weber, L. (2010). Marketing en las redes sociales. En L. Weber. Mexico: Mc Graw Hill.

Wells, Moriaty, \& Burnett. (2007). Publicidad, Principios y practicas. Mexico: Pearson. 